\title{
Towards the Investigation of Unsteady Spoiler Aerodynamics
}

\author{
Sven Geisbauer* \\ DLR, German Aerospace Center, 38108 Braunschweig, Germany \\ and \\ Thomas Löser ${ }^{\dagger}$ \\ German-Dutch Wind Tunnels (DNW), 38108 Braunschweig, Germany
}

\begin{abstract}
Aircraft configurations with deployed control surfaces are increasingly in the limelight of aerodynamic investigations using high-fidelity based Computational Fluid Dynamics to possibly reduce the conservatism in the design process. So far, most applications focus on static control surface deflections. To close the gap towards future simulations of entire flight maneuvers, including gust encounters, the dynamic motion of the control surfaces and their transient impact on the aircraft configuration need to be taken into account. Hence, extending the application range of its flow solver TAU is of strategic interest for the Institute of Aerodynamics and Flow Technology of the German Aerospace Center (DLR). To support these numerical activities DLR developed a wind tunnel model with an active spoiler. In collaboration with German-Dutch Wind Tunnels, the aerodynamic performance of this model has been examined in two wind tunnel campaigns in 2016, focusing on static and dynamic spoiler deflections. The present paper gives an overview on the motivation of this work and highlights the experimental setup as well as exemplary results. The data will later be used to further validate the DLR flow solver TAU.
\end{abstract}

\section{Introduction}

Computational Fluid Dynamics, in particular the methods based on solving the Reynolds-averaged NavierStokes (RANS) equations, nowadays have reached such a level of maturity that they have become an indispensable tool in the aerodynamic design process of any new aircraft. ${ }^{1,2}$ This holds especially for predicting the performance in the cruise design point. Benchmark results, as for example from the AIAA CFD Drag Prediction Workshop series, have shown that RANS methods are able to predict incremental differences, as long as the flow is mainly attached. ${ }^{3}$

In recent years, however, there has been a growing interest in applying RANS methods for the full flight envelope. This extension of the applications is directly related to the growing role of high-fidelity CFD in the industrial design process. CFD is no longer used for performance predictions only, but also increasingly to determine aerodynamic loads or stability and control characteristics. ${ }^{4}$ Hence, the flow conditions CFD has to cope with have become far more challenging and diverse than the traditional $M=0.85$ transonic cruise flight case. In addition, the geometries to be investigated are increasingly detailed, often featuring deployed control surfaces, high-lift systems or smaller devices such as vortex generators. It is therefore important to note that robustness or usability of steady methods no longer seem to impede their application within the entire flight envelope. ${ }^{5}$ On the other hand, it is also clear that despite the growing role of CFD in the industrial design process there is still a lack of confidence when it comes to flight conditions with significantly separated flows. ${ }^{6}$ As a consequence, the impact of turbulence modeling and the discretization approach is still subject to ongoing research, requiring validation data.

As part of its long-term digital aircraft vision the Institute of Aerodynamics and Flow Technology of the German Aerospace Center (DLR) is fostering the development of its RANS flow solver TAU to allow for the

\footnotetext{
*Research Scientist, Institute of Aerodynamics and Flow Technology, Lilienthalplatz 7, AIAA member.

${ }^{\dagger}$ Research Scientist, Low Speed Wind Tunnel Braunschweig (DNW-NWB), Lilienthalplatz 7.
} 
simulation of entire flight maneuvers, such as gust encounters. ${ }^{7}$ To pave the way it is indispensable to also take the dynamic motion of deflecting control surfaces, in particular spoilers and their transient impact on the aircraft, into account. Today, this still is a challenging task and it needs to be ensured that the unsteady aerodynamic effects are properly resolved, both spatially and temporarily.

In order to support these numerical activities, the work presented in this paper aims at acquiring experimental data to validate the DLR flow solver TAU. As DLR was lacking a wind tunnel model with the desired functionality of a rapidly moving spoiler, it was decided to modify an existing, large-scale wind tunnel model by integrating a dynamic spoiler panel. Hence, the general characteristics of static spoilers as well as the influence of the relevant motion parameters, such as deflection rate or type of deflection, were investigated in this campaign.

\section{Spoiler Applications and Spoiler Aerodynamics}

Spoilers are multifunctional control devices on the upper wing surface, serving a variety of tasks in the flight control system of modern airliners. Their primary task is to act as an inflight speed brake by inducing a controlled flow separation and thus reducing the lift and increasing the drag. This "spoiling" of the flow over the wing allows for higher sink rates at a given airspeed. Although they are temporarily used on nearly every flight to control the vertical flight path, they are designed such to enable the aircraft to conduct an emergency descent with very high sink rates, as required for instance after a failure of the aircraft pressurization system. Above that, they assist the ailerons in roll control and are used as lift dumpers after landing to help reducing the landing distance.

Since the advent of the active control technology (ACT) in the late 1970's spoilers and ailerons perform additional tasks, such as maneuver load alleviation (MLA) and gust load alleviation (GLA), flutter suppression or enhancement of the passenger ride comfort during turbulence, effectively reducing the wing loads in those situations. $^{8-10}$ While rather low deflection rates are required for the MLA system, an effective GLA system requires much higher rates to counteract the sudden change in onflow conditions. As shown by Xu and Kroo, the integration of both MLA and GLA systems into a baseline wing of a transonic transport aircraft can lead to a reduction of the direct operating cost of nearly $6 \%$ and fuel savings of $9 \% .{ }^{11}$ According to Flaig ${ }^{6}$ the combined use of MLA and GLA technologies applied to the Airbus A380 led to a reduction of wing root bending moment (WRBM) of $20 \%$. In a recent DLR study it was demonstrated that WRBM savings of $30 \%$ can be achieved if an optimized aileron geometry is used. ${ }^{12}$

Especially for gust critical wing designs, spoilers are applied as they allow for a fast (re-)distribution of the aerodynamic loads due to their high actuation rates, turning them into the backbone of modern GLA systems.

But despite this multitude of tasks assigned to spoilers, their performance is difficult to predict for numerical methods as they exhibit a strongly nonlinear aerodynamic behavior, in contrast to ailerons. Considering dynamic deflections, additional unsteady effects, such as the adverse lift, i. e. a short-term lift augmentation as illustrated in Fig. 1, might occur. A lot of efforts were therefore undertaken to gain an understanding of the relevant flow physics around control surfaces, in particular spoilers. An overview is given in the following.

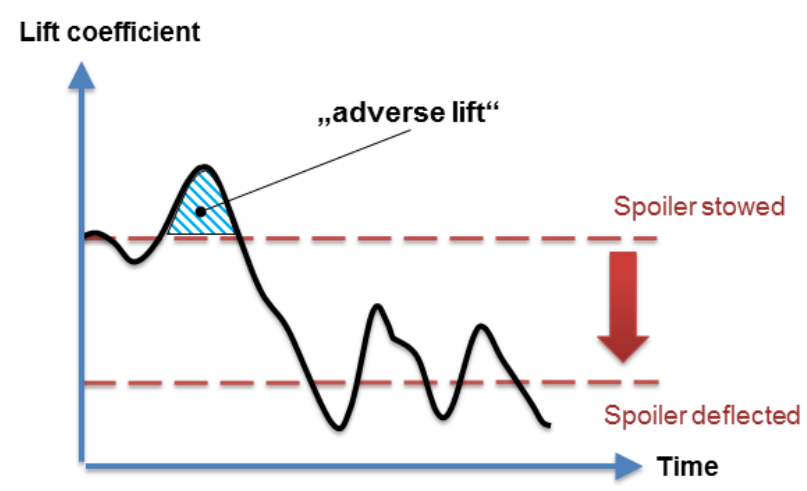

Figure 1. Short-term lift augmentation (adverse lift) on a rapidly deployed spoiler. 


\section{II.A. Literature Review on Experimental Spoiler Aerodynamics}

In 1927, Fage and Johansen ${ }^{13}$ conducted wind tunnel experiments to investigate the vortex systems behind an inclined flat plate. They found out that the shedding frequency of the vortices behind the plate was directly proportional to the onflow velocity, but inversely proportional to the inclination of the plate and, consequently, the width of the wake. In addition, they discovered that the characteristic length of the plate for forming the nondimensional shedding frequency or Strouhal number was its chord multiplied with the sine of its incidence. Based on their findings for the flat plate, the term "spoiler projection height" was later introduced and is now widely accepted as the characteristic length of a spoiler, e. g. when forming the vortex shedding Strouhal number. It therefore certainly is legitimate to state that their work is a significant contribution to the birth of experimental spoiler aerodynamics.

The majority of the research activities however did not begin until the late 1970's, initially focusing on the mean and unsteady surface pressure and wake velocity distributions at airfoils with statically deflected twodimensional spoilers. While most of these experimental activities aimed at gaining a better understanding of the nonlinearities and dead bands associated to spoilers and their causes, others already tried to validate first numerical methods with this data. Mack et al. ${ }^{14}$ found out that the spoiler effectiveness, i. e. the incremental lift loss with increasing deflection, is nonlinear for clean configurations with retracted leading and trailing edge devices. The nonlinearities got more pronounced if the trailing edge flaps are deployed. These nonlinearities, and the fact that they were hardly predictable at that time, made them state that the aerodynamic characteristics of spoilers were "the most difficult to predict of any control devices".

In the following years, researchers ${ }^{15-18}$ continued to investigate the unsteady flow patterns which are caused by an interaction of shed vortices from the spoiler tip and the airfoil trailing edge. By analyzing surface pressure distributions, boundary layer profiles or wake velocity distributions they confirmed the findings by Fage and Johansen that the vortex shedding frequency was inversely proportional to the spoiler deflection. Moreover, it became clear that the vortex energy was concentrated in a narrow frequency band and that the bandwidth became narrower the more the spoiler had been deflected.

Wentz et al. ${ }^{19}$ were among the first to investigate the impact of the spoiler geometry on its aerodynamic characteristics. They found out that the relevant parameter to determine spoiler control effectiveness was its projection height, i. e. the vertical distance from the spoiler tip to the trailing edge of the airfoil, rather than its chord or deflection angle. This essentially confirmed the earlier findings of Fage and Johansen. In addition, it turned out that by certain modifications of the spoiler geometry, such as hinge line gaps, porosity or trailing edge modifications, the maximum control effectiveness was reduced. However, it was possible to reduce typical dead band characteristics or peak frequencies in the wake with those modifications. Their work therefore belongs to the first contributions in the field of reducing the adverse spoiler effects.

A comprehensive study is presented by Siddalingappa and Hancock ${ }^{20}$. They studied 2D and 3D spoiler effects, interactions with a deployed flap and compared the characteristics of spoilers with the ones of ailerons. Moreover, they assessed compressibility effects on spoilers and ailerons and discussed the transient lag effects during spoiler deployment. They observed that the lag effects increased the more forward the spoiler hinge line was located. Additionally, during fast spoiler deployment experiments they noticed an initial lift overshoot, a phenomenon which will be known as adverse lift effect and studied in more detail in the years to come. At that stage, however, they did not further attempt to measure the aerodynamic delays or to explain the aerodynamic effects causing it. As far as 3D spoilers are concerned the spoiler effectivity was augmented compared to the $2 \mathrm{D}$ spoiler. The reason for that consisted in the interaction of the vortices from spoiler tip and edges, which effectively reduced the reattachment length behind the spoiler. The reduced reattachment length in turn meant that the curvature of the dividing streamlines had increased, leading to a higher suction pressure within the separated flow region and, thus, a more efficient spoiler.

The research conducted by Hancock ${ }^{21}$ and Mabey ${ }^{22}$ specifically addresses the dynamic effects of controls. Hancock qualitatively described the physical effects during spoiler deployment, which eventually led to the already mentioned time lags as soon as the spoiler was rapidly moved. He also stated that there was a hysteresis effect, as the lag effects during spoiler deployment had not been observed during its retraction. Hancock however did not comment on how fast a deflection of the spoiler needed to be to be considered rapid.

Mabey, in contrast, tried to quantify that by introducing the nondimensional time parameter $t^{*}$ as defined in Eq. (1), with $c$ being the chord length of the airfoil and $V_{\infty}$ the undisturbed onflow velocity.

$$
t^{*}=t \frac{V_{\infty}}{c}
$$


He stated that a deflection was considered rapid if the spoiler reached its final position in $t^{*} \leq 5$. In that case, a "significant" initial lift overshoot, which he called adverse lift, would be observed.

Equation (1) also indicates the scaling requirements for conducting wind tunnel tests, as $t^{*}$ is the relevant similarity parameter. For instance, if a spoiler reaches its final position after $t=1 \mathrm{~s}$ at full scale and a given onflow velocity, the same aerodynamic behavior for a 1:10 model at the given velocity is observed only if the model spoiler reaches its end position after $t=0.1 \mathrm{~s}$. It easily becomes apparent that very high actuation rates have to be achieved if the wind tunnel results shall be transferred to full scale problems.

Beginning of the mid 1980's the interest of the research groups shifted from static to moving spoilers. Consigny et al. ${ }^{23}$ investigated harmonically oscillating spoilers as well as rapidly deflecting spoilers. They confirmed the strong hysteresis already mentioned by Hancock. In contrast to Mabey's findings, he even observed adverse lift for deployment times up to $t^{*}=9$.

Kalligas $^{24}$ conducted an extensive experimental campaign to analyze the adverse lift and its influential parameters. He was one of the first to not only examine the lift response, but also the drag response, which showed an even more sluggish behavior. In his work, he did not discover adverse lift effects for deployment times of $t^{*}>8$.

Costes et al. ${ }^{25}$ were focusing on harmonic oscillations and ramp-type deflections of a spoiler and a flap. They assessed the mutual interference as well as Reynolds number effects. They found out that the nonlinearities of a rapidly deploying spoiler increased with deflection rate and that no adverse effects were observed for a rapidly moving flap. The Reynolds number effects in their study were considered weak.

Nelson et al. ${ }^{26}$ also investigated the harmonically-oscillating and ramp-type spoiler. The focus in their work consisted in examining the vortex development and vorticity transport by visualizing the wake structures using laser-doppler anemometry.

While previous researchers focused on understanding the flow physics related to static and dynamic spoilers, Yeung et al. ${ }^{27}$ focused on the adverse lift phenomenon and tried to abate it with base-venting, i. e. by introducing a gap at the spoiler hinge line. They successfully demonstrated that the adverse aerodynamic effects could be mitigated through base-venting, effectively proving the earlier results by Wentz et al.

The work by Mineck ${ }^{28}$ is the only one aware to the authors in which the performance of spoilers and ailerons was investigated up to flight Reynolds numbers of 30 million and in transonic conditions. He found out that the spoiler effectiveness, measured in terms of increment of rolling moment coefficient, was mostly increased when moving from low Reynolds numbers of 3 million up to 22 million. A further Reynolds number increase up to 30 million led to small changes only.

Finally, it needs to be mentioned that the former Research and Technology Organization of NATO published an extensive data base for verification and validation of unsteady computational methods. ${ }^{29}$ Among those is a test case for the so-called Benchmark Active Controls Technology (BACT) model by NASA Langley, including experimental data for a simple rectangular wing with static and harmonically-oscillating spoiler, up to transonic flow conditions. It is well documented and the geometry is publicly available, but unfortunately it does not include any data on ramp-type spoiler deflections. Nonetheless, it was used at DLR in a previous work to start validating its flow solver TAU for cases with static spoiler deflections in subsonic and transonic conditions.

\section{II.A.1. Summary and Conclusions}

The researchers have used different techniques, like oil flow visualization or laser-doppler anemometry, static pressure probes and unsteady surface pressure transducers, accelerometers as well as hot-wire anemometry, to gather the data of the mainly two-dimensional test cases. With few exceptions, the experiments were performed at Reynolds numbers below one million, based on the chord length of the airfoil. Dynamic spoilers were mostly driven by a hydraulic actuation system.

The literature review also revealed that the investigated configurations are diverse and often not publicly available or not available in a sufficient quality for further CFD investigations. In addition, test conditions were sometimes not fully known or documented. Moreover, it became clear that the onset of adverse lift was observed up to nondimensional deployment times of $t^{*}=9$, indicating that the findings of Mabey $\left(t^{*}=5\right)$ seem to be very conservative.

For those reasons, and fully aware of the vast amount of information presented in the work of the aforementioned researchers, DLR decided to develop its own wind tunnel model, conduct its own tests in collaboration with the German-Dutch Wind Tunnels and use this data at a later stage for the validation of its in-house flow solver TAU. 


\section{Experimental Setup}

Two wind tunnel campaigns were conducted in 2016 to investigate the aerodynamic characteristics of static and dynamic spoiler deflections. Within the first campaign, the one presented in this paper, the focus laid on collecting steady and unsteady surface pressure data. The key aspect in the second campaign was to visualize the flow field around static and dynamic spoilers, applying time-accurate particle image velocimetry. The results of the second campaign are not covered in this paper, but will be published at a later stage.

\section{III.A. The DLR-F15DS Wind Tunnel Model}

The baseline DLR-F15 model is a 2D wall-to-wall high-lift model with a constant chord of $c=600 \mathrm{~mm}$ and a span of $b=2800 \mathrm{~mm}$. Its leading and trailing edges may be exchanged, while the main wing section is always the same. Thus, it allows for investigating 1-element up to 3-element high-lift configurations. The clean wing geometry is taken from a generic transport aircraft research configuration and features a state of the art transonic turbulent airfoil. ${ }^{30}$

The wind tunnel model used in this work, the DLR-F15DS (dynamic spoiler) model, is a derivative of the baseline DLR-F15 model. For the very first time in working with the F15 model the clean wing geometry is examined, i. e. no leading or trailing edge devices were deployed. An all-new trailing edge module therefore was developed and manufactured, featuring a dynamic spoiler panel hinged at $x / c=0.745$, as shown in Fig. 2. The center section of the spoiler coincides with the center section of the airfoil. The spoiler has a chord length of $c_{s}=90 \mathrm{~mm}$ and a span of $b_{s}=600 \mathrm{~mm}$, leading to a spoiler aspect ratio of $\mathrm{AR}_{s}=6.7$. An overview on the components of the new trailing edge module and on the configuration in fully assembled state, partially with wind tunnel components, is shown in Fig. 3.

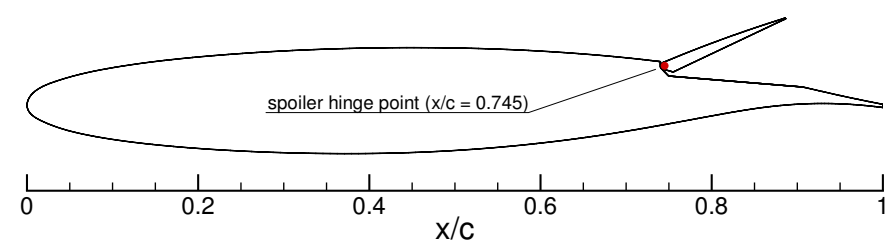

Figure 2. The DLR-F15 geometry in clean configuration with deployed spoiler.

The DLR-F15DS model is equipped with about 150 static pressure taps. The center section offers the densest distribution of pressure probes, while there are also two less dense distributions located symmetrically to the center section, close to the wind tunnel walls. They serve for examining the two-dimensionality of the flow. In addition, 30 unsteady pressure transducers are distributed in the center section of the airfoil. Hence, for static spoiler deflections surface pressure information in the center section is gathered at more than 100 positions. Four unsteady pressure transducers are located on the spoiler, three on its front face and one on its back face. Additionally, there are four pressure taps for static measurements on the front face of the spoiler.

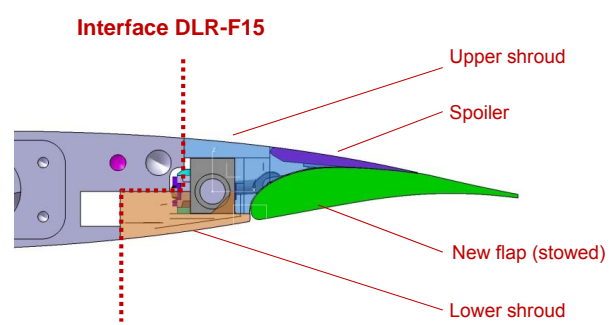

(a) The new trailing edge module.

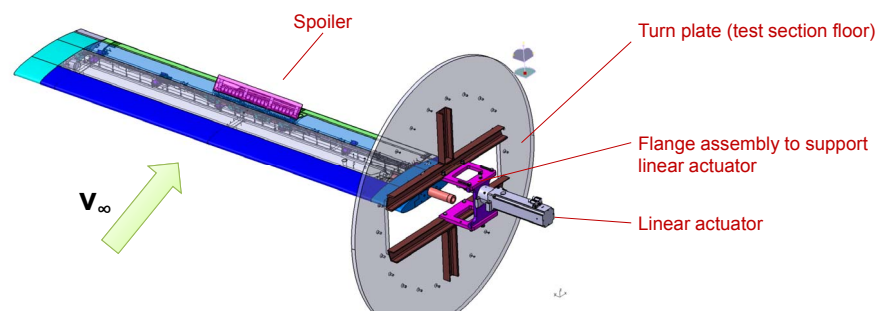

(b) Assembly with actuator position. Unlike shown, the model is mounted vertically in the wind tunnel.

Figure 3. Overview on the CAD geometry of the DLR-F15DS model.

The spoiler is actuated by an electrically driven linear actuator, a compact unit consisting of a brushless servo motor with ball screw, encoder and holding brake. Due to lack of space it was not possible to integrate 
an adequately sized drive directly into the model. Further constraints consisted in the fact that the model needed to be mounted vertically in the test section of the wind tunnel to achieve the aforementioned 2D wall-to-wall configuration. However, on top of that, the model can also be mounted in such a way that it is supported from the floor of the test section only, turning it into a $2.5 \mathrm{D}$ model which allows for investigating sweep effects. Although this 2.5D assembly of the DLR-F15DS model was not investigated yet, the possibility to do so at a later stage was a key aspect for the development of the spoiler kinematics. Hence, it was decided that the spoiler should be driven from the lower side only. For those reasons, it is located outside of the test section, as depicted in Fig. 3(b).

During the concept finding phase for the kinematics it additionally turned out that, with the available space within the model and a distance of around $2 \mathrm{~m}$ to be covered from the actuator to the spoiler, a torque drive was not feasible due insufficient torsional stiffness. As a consequence a push rod, driven by the linear motion of the actuator, was chosen. Four levers distributed along the spoiler span turn the translational motion of the rod into a rotational motion of the spoiler. Threaded bars in between those levers allow for an adjustment of the lever position by hand. It can thus be ensured that the translational motion of the push rod is evenly transformed into a rotational motion of the spoiler, preventing a torsion of the spoiler during deployment. A close-up view on the internal spoiler kinematics is shown in Fig. 4.

Another key aspect of the construction was to try to reduce the number of mechanical components in the kinematics to help minimizing the mechanical play. For this reason and also due to the space constraints, only a small translational motion of $23 \mathrm{~mm}$ is required to fully deploy the spoiler into its $90^{\circ}$ position from fully retracted state. Hence, the entire actuation system including motion controller had to be very precise in order not to damage the model.

A further advantage of the DLR-F15DS model is the fact that it was constructed with materials to sustain cryogenic conditions. It is thus possible to use this model at a later stage in a cryogenic wind tunnel to investigate Reynolds number effects.

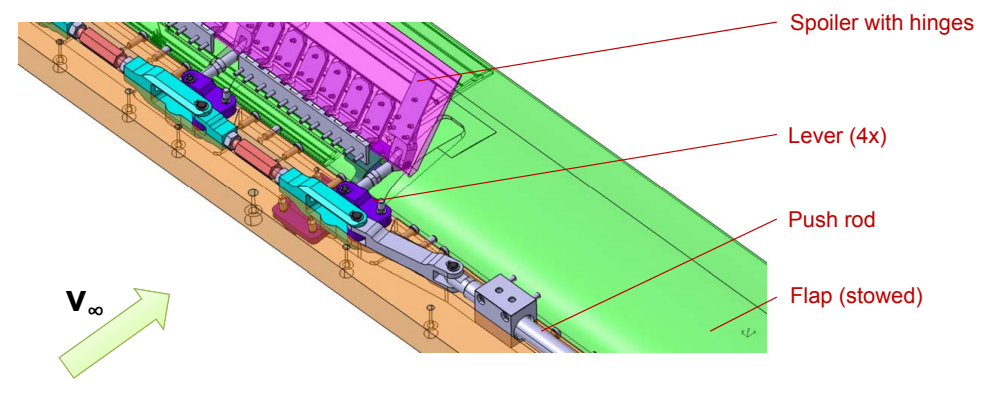

Figure 4. Internal kinematics in the trailing edge module of the DLR-F15DS model.

\section{III.B. Wind Tunnel and Test Conditions}

The tests were conducted in the low speed wind tunnel Braunschweig of the German-Dutch Wind Tunnels (DNW-NWB). The NWB is a closed return, atmospheric wind tunnel, offering a cross section of $3.25 \mathrm{~m} \mathrm{x}$ $2.80 \mathrm{~m}$. The experiments were conducted in the closed test section, in which a maximum undisturbed onflow velocity of $v_{\infty}=90 \mathrm{~m} \mathrm{~s}^{-1}$ can be achieved. During the experiments the maximum onflow Mach number was $M_{\infty}=0.2$ or $v_{\infty} \approx 69 \mathrm{~m} \mathrm{~s}^{-1}$. The corresponding Reynolds number amounts to $R e_{c}=2,700,000$. Transition was fixed at the leading edge of the model at $x / c=0$, using an adhesive tape of $0.2 \mathrm{~mm}$ height and $3 \mathrm{~mm}$ width. With fully deployed spoiler at $\delta=90^{\circ}$ the maximum blockage in the test section was $4 \%$ at $\alpha=10^{\circ}$.

The configuration has been investigated clean, i. e. without any high-lift devices, and with static spoiler deflections of up to $85^{\circ}$, up to the maximum lift region of the airfoil. In addition, two dynamic scenarios were examined subsequently as shown in Fig. 5:

- Scenario (1) is a ramp-type deflection of the spoiler, always from fully retracted state. The motion is described by the time and deployment parameters depicted in Fig. 5(a).

- Scenario (2) consists in a harmonic motion of the spoiler, based on a sinusoidal motion with varying frequency and amplitude around a predeflected spoiler. The motion is defined by the input parameters shown in Fig. 5(b). 
The spoiler could be remotely-controlled either in manual mode or in automatic mode. In automatic mode, the parameters for the motion profiles as shown in Fig. 5 are preset, the motion is then automatically triggered by an external TTL-signal. Manual mode was only used to couple the actuator to the kinematics, all experimental runs were conducted in automatic mode. The majority of the runs was conducted in scenario (1), in order to investigate the unsteady spoiler effects such as adverse lift. After conducting $\alpha$-sweep runs for various static spoiler deflections, the deployment time $t_{\text {deploy }}$ of the spoiler as depicted in Fig. 5(a) has been gradually reduced, towards the limits of the actuator. The investigated parameter range is summarized in Table 1. Figure 6 shows the vertical assembly of the DLR-F15DS model in the closed test section of the DNW-NWB wind tunnel.

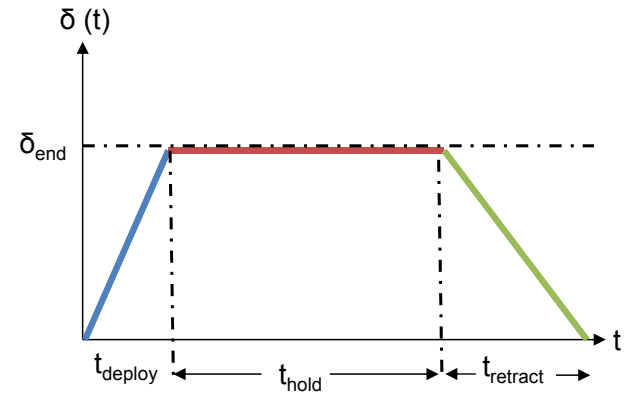

(a) Scenario (1): ramp-type deflection.

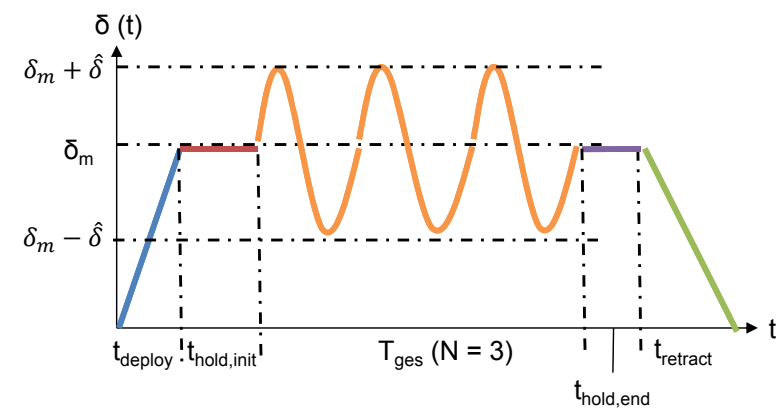

(b) Scenario (2): harmonic oscillation about mean deflection.

Figure 5. Schematic spoiler motion for the dynamic cases.

Table 1. Overview on the performed experimental runs.

\begin{tabular}{lc}
\hline \hline Parameter & Range \\
\hline Mach number & $0.1,0.15,0.2$ \\
Corresponding Reynolds numbers & $1,300,000-2,700,000$ \\
Angle of attack (clean and static deflections) & $-5^{\circ}$ to $15^{\circ}$ \\
Angle of attack (dynamic deflections) & $-5^{\circ}, 0^{\circ}, 5^{\circ}, 10^{\circ}$ \\
Static spoiler deflection angles & $0^{\circ}, 2.5^{\circ}, 5^{\circ}, 10^{\circ}$ to $80^{\circ}$ with $\Delta \delta=10^{\circ}, 85^{\circ}$ \\
Dynamic spoiler deflection angles & $10^{\circ}, 30^{\circ}, 50^{\circ}, 70^{\circ}$ \\
Spoiler deployment times & $60 \mathrm{~ms}$ to $500 \mathrm{~ms}$ \\
Reduced deployment time $t^{*}=\frac{t_{\text {deploy }} \cdot v_{\infty}}{c}$ & 6.8 to 57.4 \\
Frequency $f$ for harmonic spoiler motion & $0 \mathrm{~Hz}$ to $10 \mathrm{~Hz}$, depending on amplitude \\
Corresponding reduced frequency $k$, based on spoiler chord & 0.016 to 0.16 \\
Amplitudes & $5^{\circ}$ to $20^{\circ}$ \\
\hline \hline
\end{tabular}

\section{III.C. Model Instrumentation and Data Acquisition}

The following data were recorded during the experiments:

- the static pressures using 147 pressure taps and PSI modules, distributed along the center section and two outer sections,

- the signals of 30 unsteady pressure transducers distributed along the center section,

- the position of the push rod using a fully-integrated, inductive and absolute position transducer and

- the position of the spoiler using an external 3D video tracking system, using stereo pattern recognition (SPR) to track the spoiler motion.

The signals of the unsteady pressure transducers were sampled at $1,000 \mathrm{~Hz}$ and low-pass filtered with a cutoff frequency of $391 \mathrm{~Hz}$ before analog-to-digital conversion, using a 16-bit, 48 channel transient recorder. 


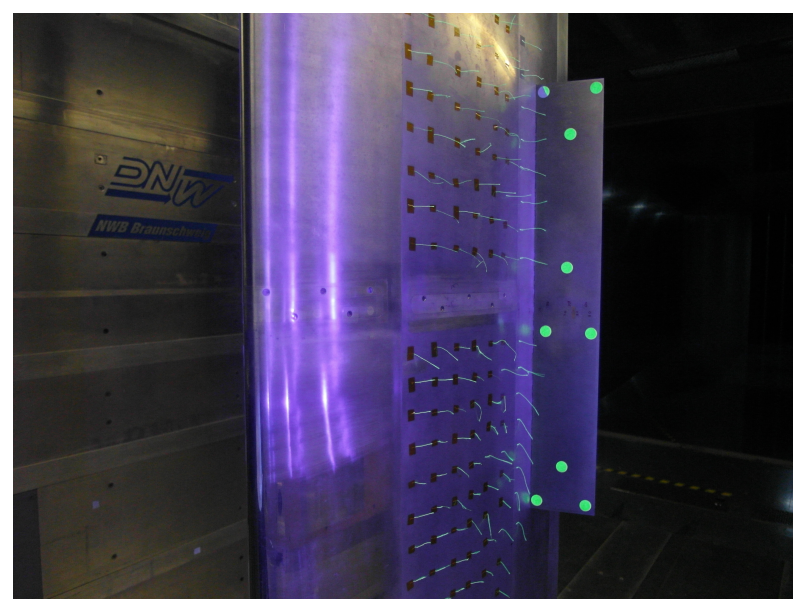

(a) Front view on deployed spoiler with SPR markers and tufts in ultraviolet light.

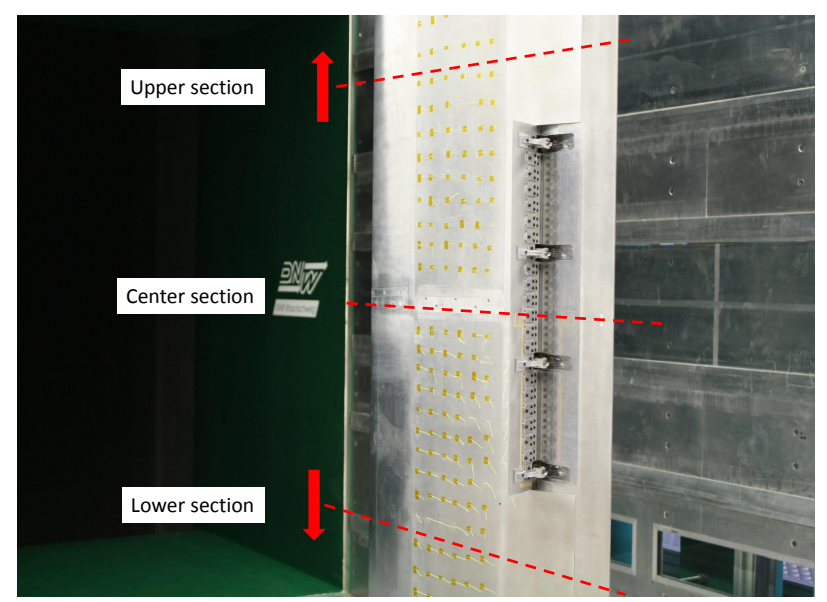

(b) Rear view on deployed spoiler, lever kinematics and pressure sections. The upper and lower sections, however, are further away from the center section as illustrated here.

Figure 6. Details of the DLR-F15DS model in the closed test section of the DNW-NWB wind tunnel.

The SPR video tracking system consisted of two high-speed cameras, working at a resolution of 2,336 $\mathrm{x}$ 896 pixels and a frame rate of 1,000 fps. Nine circular, fluorescent markers, as shown in Fig. 6(a), have been applied to the front face of the spoiler, close to its side edges and in its center section. The SPR system computes the centroids of every marker triangle. Based on the change of orientation of every centroid, the calibrated system can then determine the spoiler deflection angle.

Before recording the unsteady data the signals of the pressure transducers and the SPR system needed to be synchronized. This was achieved by generating a TTL-high-signal for the transient recorder and a shortterm illumination of a light emitting diode mounted in the test section for the SPR system. In addition, the TTL-signal was used to launch the spoiler motion in automatic mode. The data was recorded over $2 \mathrm{~s}$ for static spoiler measurements and over $24 \mathrm{~s}$ for dynamic measurements. Within this time window of $24 \mathrm{~s}$ four to seven repetitions have been conducted.

\section{III.D. Wind Tunnel Corrections}

The method of Allen and Vincenti, as described in Ref. 31, was applied to correct the experimental data for static cases of the clean configuration. Regarding solid and wake blockage corrections, it turned out though that the differences between corrected and uncorrected onflow velocity was below $1 \%$. Considering the blockage due to lift, the method of the same authors leads to adjustments of the angle of attack of $\Delta \alpha<0.05^{\circ}$.

On top of this "conventional" correction method the method of Maskell ${ }^{32}$ for bluff body blockage effects has been applied to determine the correction for the dynamic pressure. Similar to the first method, it yields a $1 \%$ increase in dynamic pressure. Overall, the effects due to blockage are small.

\section{III.E. Integration of the Pressure Coefficient}

The DLR-F15DS model was not equipped with a wind tunnel balance to capture the integral forces and moments. Therefore, a post-processing step was required to integrate the pressure distribution to obtain the sectional force and moment coefficients. For static spoiler deflections the distribution of the steady pressure ports as shown in Fig. 7 was used. A linear integration between the data points is performed, based on the following assumptions:

1. the model was equipped with four static pressure ports on the spoiler front face, however no information was available in the vicinity of the spoiler hinge line or its trailing edge. Therefore, two virtual pressure ports were introduced at those locations to achieve a better resolution of the actual geometry and, thus, a more reasonable integration result. Their $C_{p}$-values were assumed to be equal to the ones of the closest actual spoiler pressure port, 
2. the model was equipped with only one unsteady pressure transducer at the rear face of the spoiler. Again, two virtual pressure ports were introduced close to the hinge line and tip for the same reasons. The $C_{p}$-value of the transducer was then assigned to the two virtual ports, in fact leading to a constant pressure distribution along the spoiler rear face. Corresponding CFD studies have proved that this procedure is a valid assumption.

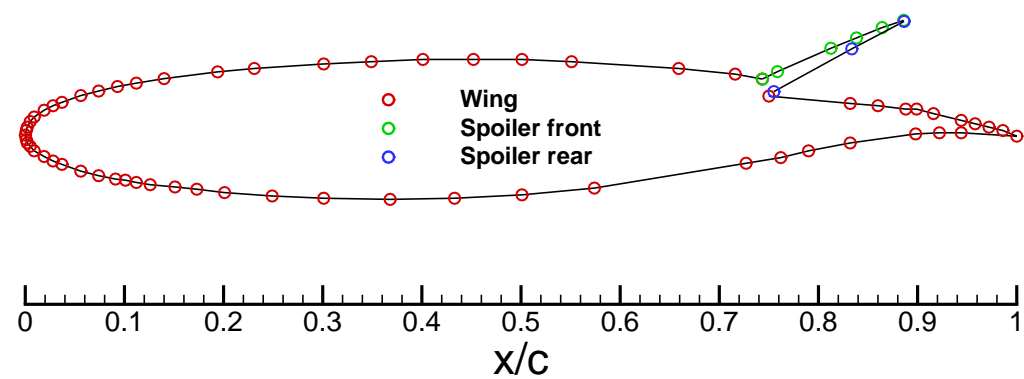

Figure 7. Distribution of the static pressure ports used for the integration of $C_{p}$.

\section{III.F. Accuracy Considerations}

\section{III.F.1. Pressure Sensing System}

The accuracy of the PSI modules and the unsteady pressure transducers amounts to $0.1 \%$ full scale (FS) output, according to the manufacturers. While the wind tunnel operator confirmed the former value, the accuracy of the unsteady transducers is more likely in the range of $0.3 \%$ FS. The accuracy of the different PSI modules and pressure transducers in capturing $C_{p}$ is summarized in Table 2 for the most relevant flow condition of $M=0.2$.

Table 2. Estimation of the measurement accuracy for the surface pressure coefficient at $M=0.2$.

\begin{tabular}{lcc}
\hline \hline Type & Accuracy & $\Delta C_{p}$ \\
\hline Static pressure ports / PSI modules & $0.1 \%$ FS & $0.01-0.04$ \\
Unsteady pressure transducers & $0.3 \%$ FS & $0.04-0.11$ \\
\hline \hline
\end{tabular}

\section{III.F.2. Positioning of Linear Actuator}

The chosen kinematic setup leads to a nonlinear relation between the displacement of the linear actuator and the spoiler deflection. This relation was predetermined using the parametric CAD model of the kinematics and has been implemented in the motion controller of the actuator. Due to the fact that the "real" kinematics differ from the idealized CAD representation, for instance due to mechanical play between the components or due to mechanical constraints imposed on the kinematics during assembly, a calibration of the linear actuator was required. The actual spoiler angle was manually measured in fully assembled state of the model and wind-off conditions, using a high-precision angle gage.

The relation between the nominal spoiler angle and the actual angle, in terms of its offset to the nominal value, is depicted in Fig. 8. Prior to calibration quite significant differences between nominal and actual spoiler angle were observed. For low and medium deflection angles, up to $\delta=50^{\circ}$, a fairly constant offset in the range of $-1.7^{\circ}$ to $-2.2^{\circ}$ was observed. As soon as the spoiler is deflected further, the offset increases linearly with deflection angle. It reached a maximum of $-4.2^{\circ}$, i. e. instead of the nominal value of $\delta=85^{\circ}$ an actual angle of $\delta=80.8^{\circ}$ was achieved. After calibration, the offset is nearly independent of the target spoiler angle, with a maximum of $-0.4^{\circ}$ at $\delta=80^{\circ}$. The mean offset, i. e. the positioning accuracy of the calibrated linear actuator, amounts to $\pm 0.2^{\circ}$.

A final check was conducted at the end of the campaign to examine if the mechanical play has changed, especially due to the dynamic tests. As shown, the curve has shifted over a wide deflection range towards 
more positive offset levels, indicating that the actual spoiler angle has again become larger than it was supposed to be and than it was immediately after the calibration. The mean offset has nearly doubled to $\pm 0.4^{\circ}$.

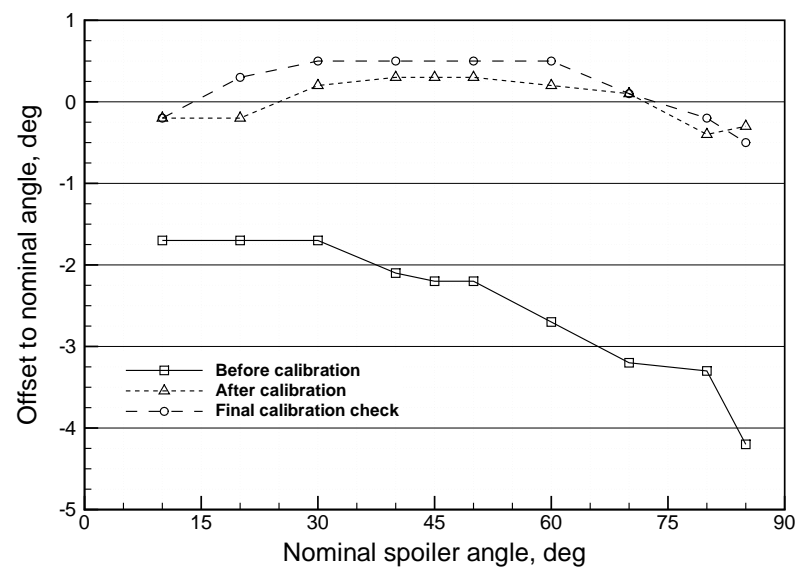

Figure 8. Accuracy of the linear actuator in positioning the spoiler.

\section{III.F.3. SPR Video Tracking System}

Apart from the accuracy of the actuator in reaching the target deflection angle the accuracy of the SPR system is of great importance for the subsequent validation of the CFD code as it was the only reliable means to track the actual spoiler position and motion in wind-on conditions. During the calibration phase the deflection angles as computed by the SPR system have been compared to ones which have been manually measured with an angle gage. Maximum differences of $\Delta \delta=3.3^{\circ}$ were observed. Applying an additional quadratic curve fit to the SPR results significantly improved its accuracy, leading to a final accuracy of $\pm 0.5^{\circ}$ compared to angle gage result.

\section{Experimental Results}

A summary of the experimental results is given in the following, focusing on the surface pressure distribution at the mid-section for the clean configuration and static spoiler deflections. Later on, using the data of the unsteady pressure transducers, the impact of the dynamic spoiler motion will be assessed.

\section{IV.A. Basic Flow Assessment}

In a first step, it was examined if the flow about the $2 \mathrm{D}$ wall-to-wall model actually is symmetric with respect to its center section. Figure 9 shows the surface pressure distributions in the different sections at two angles of attack, for the clean configuration and one with statically deployed spoiler. Minor differences exist due to a different number of active pressure ports in the sections. In case of the deflected spoiler, however, there is one distinct difference observed on the upper surface of the outer section, at $x / c=0.9$. At that position, there are consistently higher suction pressures than at the same position of the lower section, although the geometry should be identical in both sections. The same holds for their distance to the symmetry axis of the model. Although the respective pressure port is located close to an interface of model components, potentially exposing it to geometric imperfections, it is not yet understood why this difference only occurs in case of the deflected spoiler. Apart from that position, the pressure distributions are largely identical. Hence, it can be assumed that the flow is $2 \mathrm{D}$ when the spoiler is retracted and symmetric to the center section when the spoiler is deflected.

\section{IV.B. Influence of the Static Spoiler Deflection on the Base Pressure}

The steady pressure distributions have exemplary been evaluated with respect to the base pressure behind the deflected spoiler. The data of 10 static pressure ports located in this region, see Fig. 7, are plotted over 


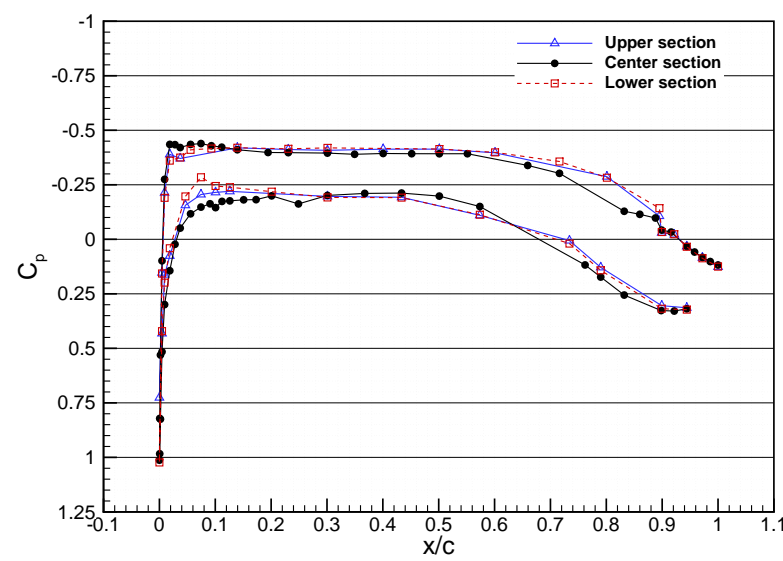

(a) $\alpha=0^{\circ}, \delta=0^{\circ}$.

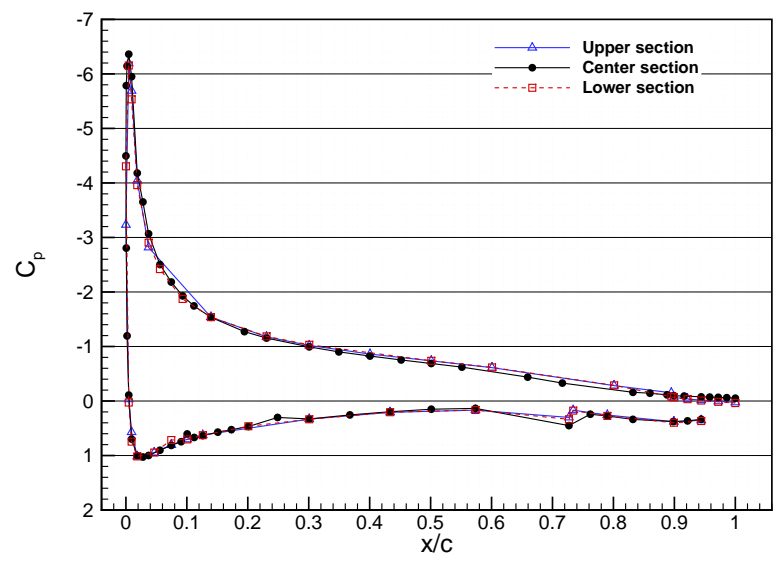

(c) $\alpha=10^{\circ}, \delta=0^{\circ}$.

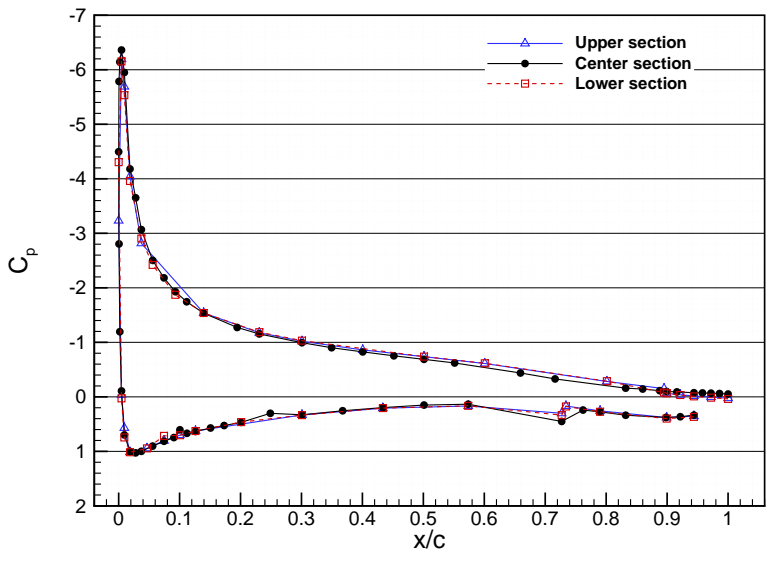

(b) $\alpha=0^{\circ}, \delta=45^{\circ}$.

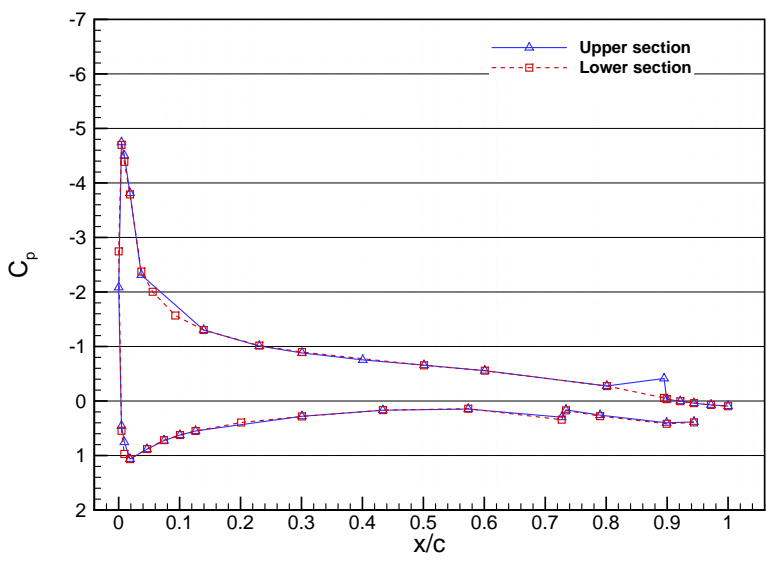

(d) $\alpha=10^{\circ}, \delta=45^{\circ}$.

Figure 9. Surface pressure distributions in the pressure sections at $M=0.2$.

$x / c$ and spoiler deflection angle as shown in Fig. 10.

At $\alpha=0^{\circ}$, Fig. 10(a), the flow does not completely detach behind the spoiler until it is deflected beyond $\delta=5^{\circ}$. Further deploying the spoiler continuously reduces the level of (now constant) base pressure, until a minimum is reached at around $\delta=70^{\circ}$ of $C_{p b}=-0.53$. When increasing the angle of attack, as shown in Fig. 10(b), the flow still reattaches until $\delta=5^{\circ}$. The minimum base pressure at $\alpha=10^{\circ}$ is nearly unchanged.

In addition to the conventional spoiler deployments Figs. 10(a) and 10(b) also include the results of a run in which the gaps along the spoiler hinge line have been sealed, using adhesive tape. As shown, the tape prevents the air from flowing through those gaps, leading to slightly lower base pressure levels. In terms of spoiler efficiency, its efficiency would be improved by closing this gap.

Figures 10(c) shows the mean base pressure, the average of the 10 signals from the static ports, over the spoiler deflection angle. Independent of the angle of attack, an increasing suction pressure behind the spoiler can be observed until a saturation is reached between $\delta=50^{\circ}-60^{\circ}$. While the base pressure changes linearly up to the saturation point for angles of attack between $-5^{\circ} \leq \alpha \leq 5^{\circ}$, a nonlinear behavior is observed at $\alpha=10^{\circ}$. The latter is probably due to the already higher aerodynamic loading of the clean configuration at this angle of attack, with potentially incipient trailing edge separation. The minimum base pressure is continuously shifted towards more negative pressure levels with increasing angle of attack. At $\alpha=10^{\circ}$, a different behavior is observed again, as the pressure levels are in between the ones from $\alpha=0^{\circ}$ and $\alpha=-5^{\circ}$.

The base pressure fluctuations derived from the static pressure ports are illustrated in Fig. 10(d). As shown the fluctuations are small and nearly constant for spoiler angles beyond $\delta=10^{\circ}$. For lower angles, there are increasing fluctuations around the mean value, with peak values reached at the lowest investigated spoiler angle of $\delta=2.5^{\circ}$. This curve nicely proves the aforementioned findings that a full flow separation behind the spoiler only occurs beyond $\delta=5^{\circ}$, which is expressed by a uniform distribution of the base 


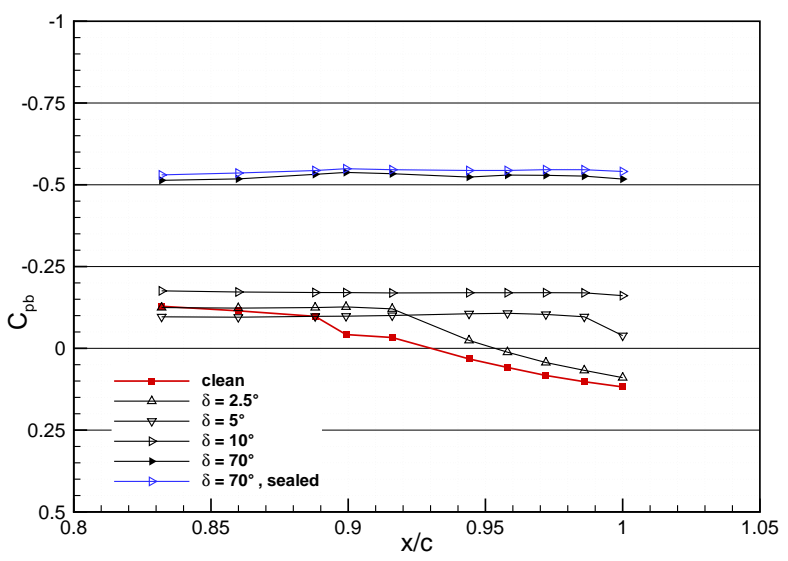

(a) $\alpha=0^{\circ}$.

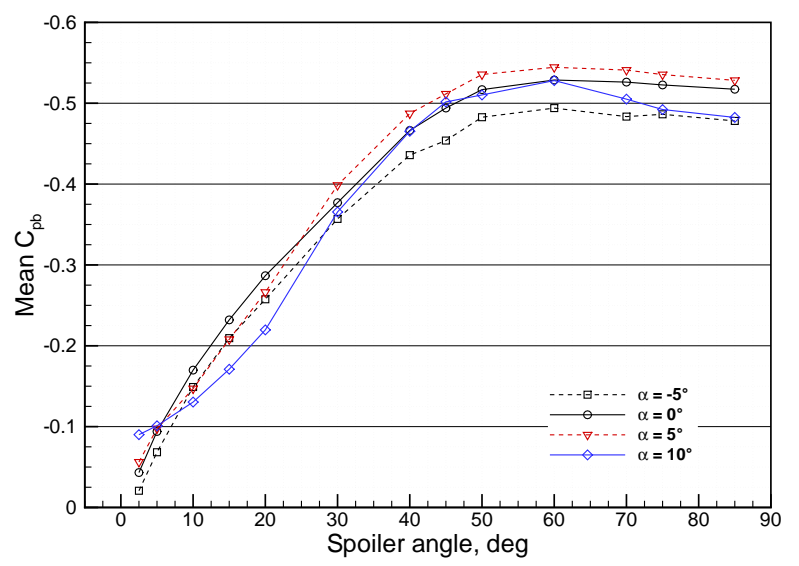

(c) Mean $C_{p b}$.

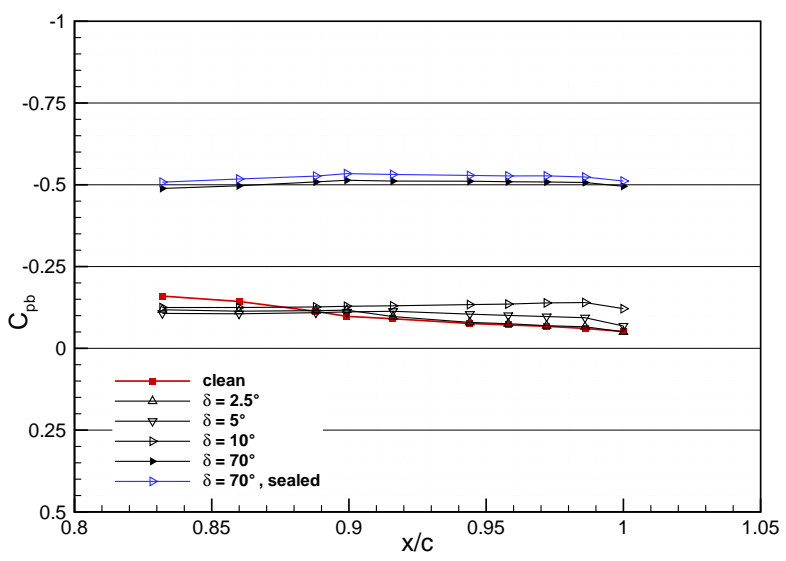

(b) $\alpha=10^{\circ}$.

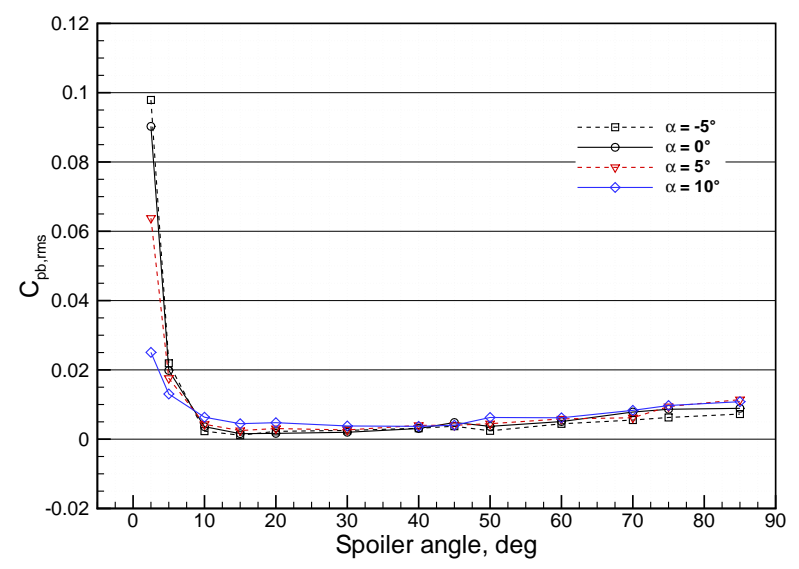

(d) RMS-values of $C_{p b}$.

Figure 10. Evaluation of the base pressure coefficient $C_{p b}$ at $M=0.2$.

pressure and, hence, low rms-values.

\section{IV.C. Influence of the Static Spoiler Deflection on the Forces and Moments}

Using the procedure described in Chapter III.E the pressure distributions of the clean configuration and the configuration with static spoiler deflections were integrated and visualized in terms of the sectional force and moment coefficients. It has to be mentioned that during the experiments an $\alpha$-sweep was conducted from $\alpha=-5^{\circ}$ up to beyond the stall angle. Having passed the stall angle the angle of attack has gradually been decreased again until $\alpha=5^{\circ}$. This was done to check for any hysteresis effects. For the sake of clarity, however, only the results of the upsweep are visualized.

\section{IV.C.1. Lift}

The impact of the spoiler on the sectional lift $c_{L}$ is shown in Fig. 11. The maximum lift coefficient is observed for the clean configuration, reaching $c_{L, \max }=1.47$ at $\alpha_{\max }=13^{\circ}$. Deploying the spoiler to $\delta=2.5^{\circ}$ shows no differences to the clean case over a wide range of angles of attack. The stall, however, is observed $1^{\circ}$ earlier. The reason for the negligible differences between the clean configuration and $\delta=2.5^{\circ}$ consists in the reattachment of the flow behind the deployed spoiler. As shown in Fig. 10(b) this is even the case at $\alpha=10^{\circ}$. Hence, although there is a small separation bubble behind the deployed spoiler, the lift almost remains unaltered. A further increase of the spoiler deflection to $\delta=20^{\circ}$ gradually reduces the lift, however still reaching the same stall angle $\alpha_{\max }=12^{\circ}$, with a sudden lift loss afterwards. The maximum lift amounts to $c_{L, \max }=1.3$, i. e. it has reduced by $12 \%$ compared to the clean configuration. 


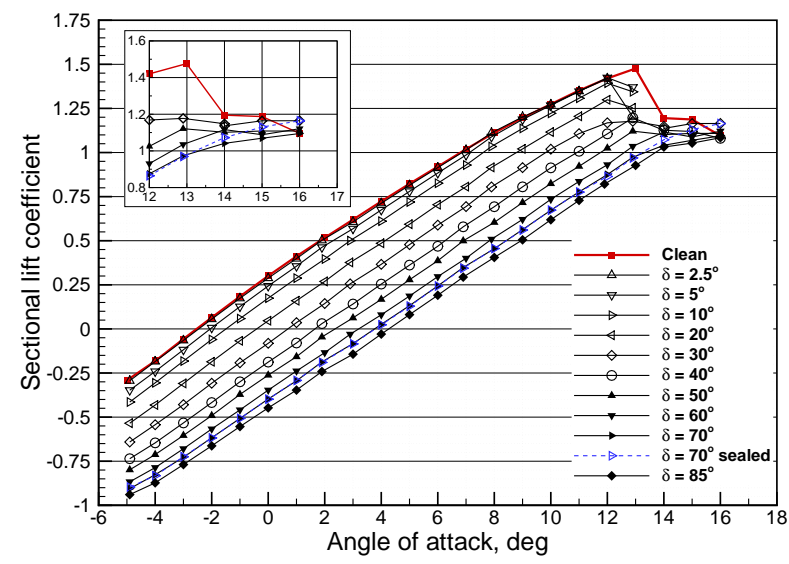

(a)

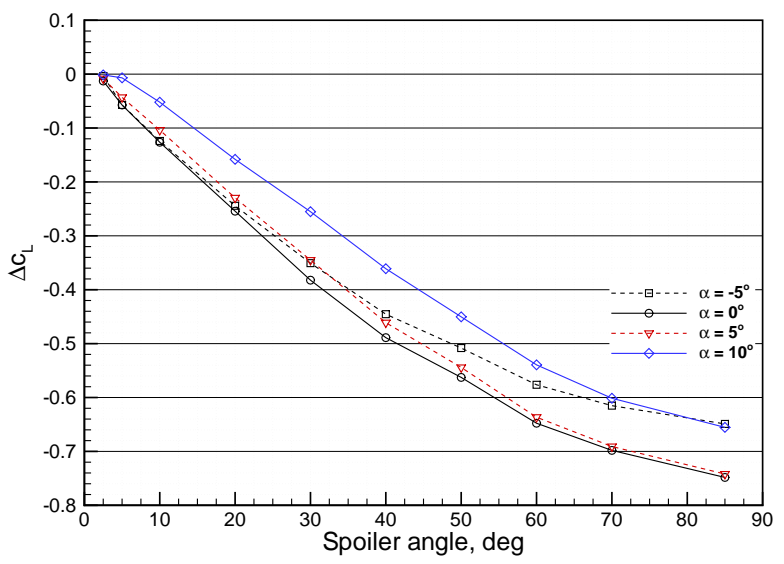

(b)

Figure 11. Influence of the static spoiler on the sectional lift at $M=0.2$.

At $\delta=30^{\circ}$ a change of the stall characteristics is observed. There no longer is a distinct stall angle or a sudden loss in lift for $\alpha>\alpha_{\max }$. Instead, a very smooth stall behavior is observed. The lift stabilizes at $c_{L, \max }$ for another $3^{\circ}$ beyond $\alpha_{\max }$. Finally, at $\alpha=16^{\circ}$, an even higher lift is observed than for the clean configuration.

At $\delta=40^{\circ}$ a similarly smooth stall behavior is observed, however the lift level is not that constant once having passed $\alpha_{\max }$. In contrast to the last case the lift never exceeds the values of the clean configuration.

For very high deployment angles beyond $40^{\circ}$, the lift at a given $\alpha$ is further reduced. At $\delta=60^{\circ}$ a maximum lift of $c_{L, \max }=1.12$ is reached at $\alpha_{\max }=14^{\circ}$. Although the maximum lift is reduced by $24 \%$ compared to the clean case, the angle of attack at which the maximum lift occurs for the first time has been increased. This behavior is even more pronounced for the two highest deflection angles at $\delta=70^{\circ}$ and $\delta=85^{\circ}$. In those cases the lift continuously increases until the end of the measured $\alpha$-range at $\alpha=16^{\circ}$, indicating that $\alpha_{\max }$ has further increased and that high spoiler deflections can delay the upstream propagation of the stall towards the leading edge.

Figure 11(b) depicts the influence of the spoiler in terms of lift loss, or spoiler effectiveness, compared to the clean configuration for a subset of angles of attack. The spoiler shows a linear behavior between $5^{\circ}<\delta<40^{\circ}$. Beyond this range, nonlinearities can be observed, for example at small angles due to the reattachment of the flow behind the spoiler and the associated ineffectiveness of the spoiler at those angles. The spoiler effectiveness is highest for $\alpha=0^{\circ}$ and $\alpha=5^{\circ}$. At $\alpha=10^{\circ}$ it is significantly reduced as the spoiler is already largely shaded by the front part of the airfoil. Hence, to receive a similar spoiler effectiveness as at the lower angles of attack, the spoiler has to be deflected further. At $\alpha=-5^{\circ}$ the effectiveness of the spoiler deteriorates at about $\delta=40^{\circ}$. It is assumed that this behavior is caused by an incipient flow separation around the trailing edge at the lower side of the airfoil.

\section{IV.C.2. Drag}

Figure 12 shows the influence of the static spoiler on the sectional drag coefficient. For the clean configuration and the configuration with $\delta=2.5^{\circ}$ small negative drag coefficients can be observed in Fig. 12(a). This is due to the fact that the drag has been computed by integrating the data of the static pressure ports, hence yielding the pressure component of the drag only and neglecting the friction part.

For deflection angles below $10^{\circ}$ and at low lift coefficients, the pressure drag is higher than the one of the clean configuration. If the lift is further increased, though, this trend is reversed. For example, if $c_{L}>0.4$ the configuration with $\delta=2.5^{\circ}$ shows a lower drag than the clean configuration. The same holds for the configurations with $\delta=5^{\circ}$ beyond $c_{L}=0.6$ and $\delta=10^{\circ}$ beyond $c_{L}=0.74$. Sealing the hinge line, as also shown in Fig. 12(a), significantly increases the drag between $-0.56<c_{L}<0.77$ and slightly reduces it beyond this range.

The relative drag increase compared to the clean configuration is shown in Fig. 12(b) for a subset of investigated angles of attack. Two observations stand out: the aforementioned drag reduction of configurations with small spoiler deflections compared to the clean configuration also becomes evident by the negative drag 


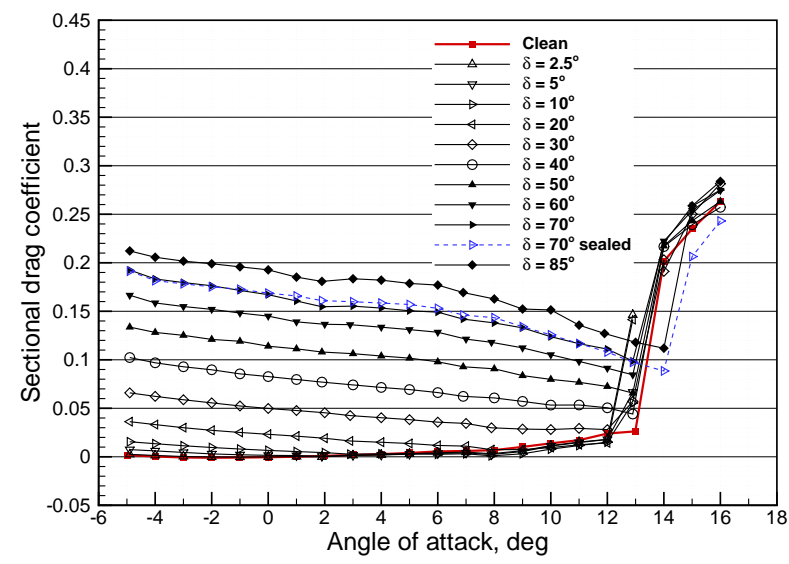

(a)

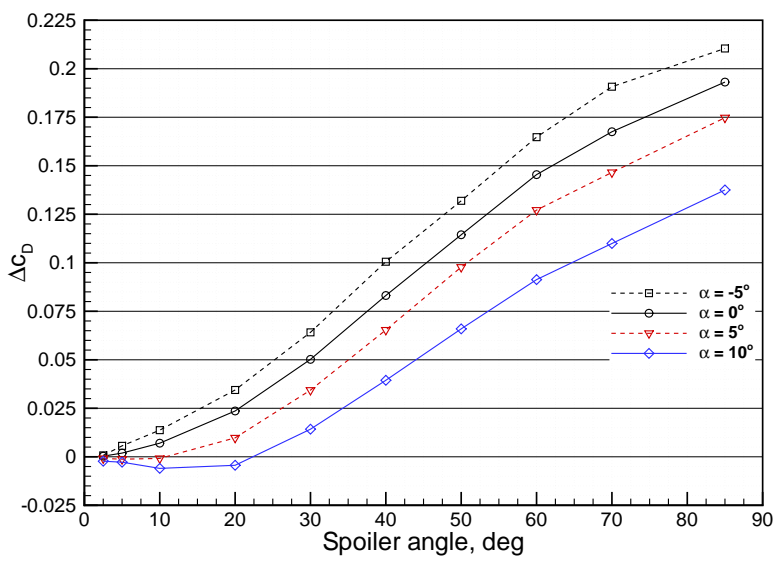

(b)

Figure 12. Influence of the static spoiler on the sectional pressure drag at $M=0.2$.

increment, especially at $\alpha=10^{\circ}$. It is not yet fully clear whether these results are caused by the integration method or if they are due to physical behavior, for instance due to delaying the upstream propagation of a trailing edge stall towards the leading edge. Further insight will be available as soon as the first computations have been conducted.

Focusing on spoiler angles beyond $\delta=20^{\circ}$, it is clear that further deflecting the spoiler always increases the drag. The drag increment at a given spoiler angle depends on the angle of attack and is highest the lower the angle of attack as the spoiler is increasingly shaded by the front part of the airfoil with increasing incidence.

\section{IV.C.3. Pitching Moment}

The spoiler influence on the sectional pitching moment coefficient is shown in Fig. 13. Starting from the clean configuration, Fig. 13(a), the pitching moment gets more positive (tail-heavy) until a maximum is reached between $\delta=60^{\circ}-70^{\circ}$. Higher deflection angles reduce the pitching moment again. Looking at the pitching moment increment in Fig. 13(b) it becomes evident that deflecting the spoiler leads to a more positive, i. e. tail-heavy, pitching moment in most investigated cases. Only the configuration with $\delta=2.5^{\circ}$ at $\alpha=-5^{\circ}$ behaves differently in that respect, indicating a small reduction of the pitching moment. The gradient of the pitching moment increment is positive until it levels out between $\delta=60^{\circ}-70^{\circ}$. For deflection angles beyond $70^{\circ}$ the gradient becomes negative.

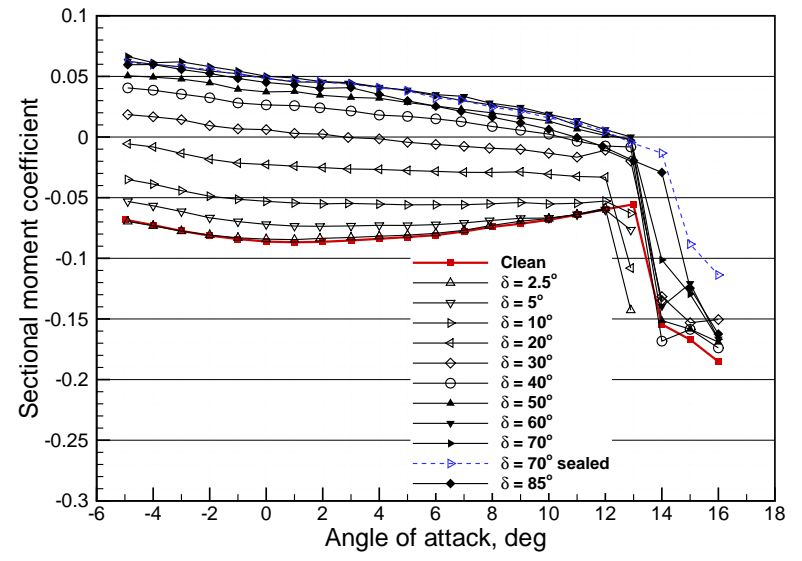

(a)

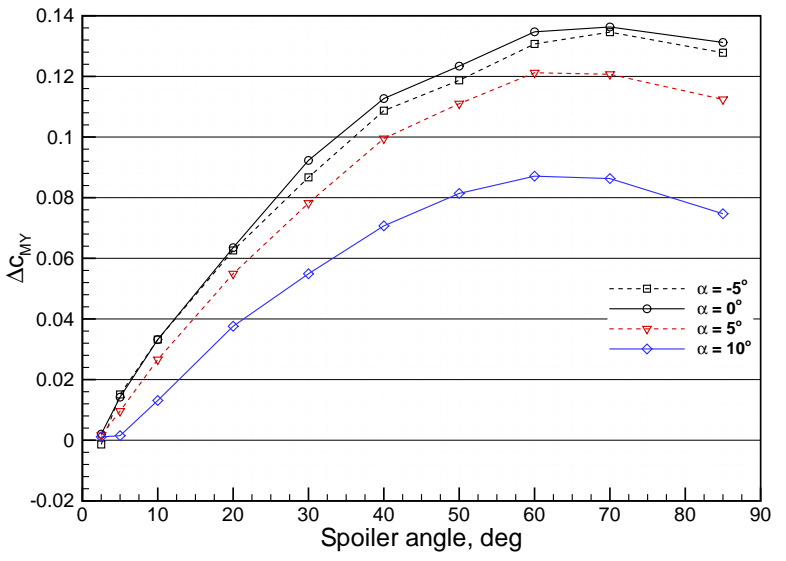

(b)

Figure 13. Influence of the static spoiler on the sectional pitching moment at $M=0.2$. 


\section{IV.D. Influence of the Static Spoiler Deflection on the Vortex Shedding}

A FFT analysis has been applied to the time-dependent signal of an unsteady pressure transducer located at $x / c=0.98$ on the upper side of the airfoil. For every statically deflected spoiler the fundamental frequency has been computed and plotted over the spoiler deflection as shown in Fig. 14. Also shown is the Strouhal number $S t_{h}$, using the spoiler projection height $h$, i. e. the vertical distance between the tip of the spoiler and the trailing edge. Due to the cutoff frequency of $391 \mathrm{~Hz}$ it was not possible to detect any dominant frequencies for spoiler angles below $\delta=20^{\circ}$.

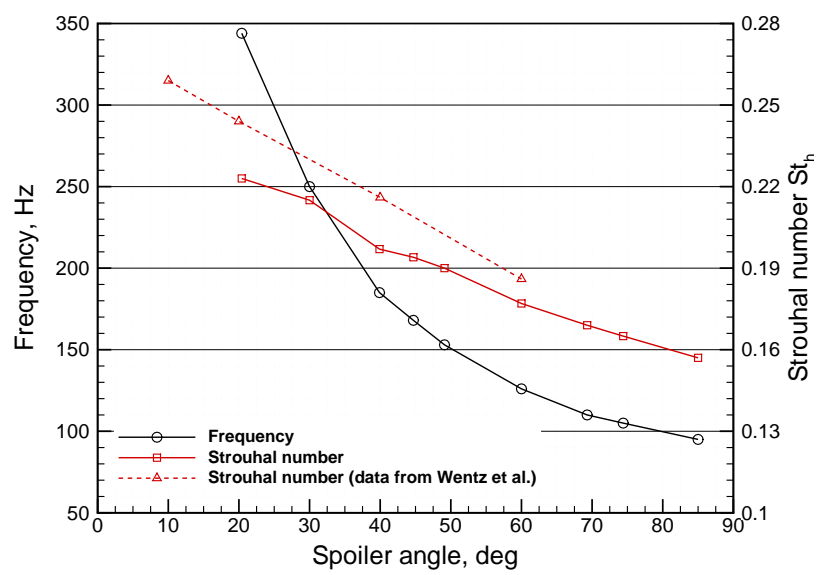

Figure 14. Influence of the static spoiler on the vortex shedding frequency at $M=0.2$.

Finally, a check was performed to assess if our approach in determining the vortex shedding frequencies based on the signals of a surface pressure transducer is feasible and leads to reasonable results. Therefore, experimental data from Wentz et al. ${ }^{19}$ is also visualized in Fig. 14. Their setup was very similar to ours, but they performed their tests at a chord Reynolds number of 2.2 million, instead of 2.7 million as in our case. In contrast to us, they determined the vortex shedding frequencies by measuring the wake velocity fluctuations, using a dual split-film anemometer. It turned out that the results in terms of Strouhal number as a function of the spoiler deflection are in good agreement.

\section{IV.E. Influence of Pressure Integration on Integrated Forces and Moments}

So far, we considered static spoiler deflections only. Hence, all pressure data or the integrated forces and moments were taken from the static pressure ports distributed along the airfoil center section, as their accuracy is superior compared to the unsteady pressure transducers. In addition, due to their larger number a much better resolution of the pressure signature around the airfoil is achieved.

When moving towards dynamic deflections, this source of information can no longer be used. Instead, only the signals of the 30 unsteady pressure transducers play a role. This in turn means that data from two different sources would have to be compared when investigating the impact of a dynamic deflection over the corresponding static deflection. Before being able to do that the influence of the two pressure sources on the pressure integration needs to be assessed in the following.

The focus of the dynamic cases consisted in investigating the transient aerodynamics during fast, ramptype spoiler deflections, according to scenario (1) shown in Fig. 5 . Only four spoiler deflection angles $\left(\delta=10^{\circ}\right.$, $\left.30^{\circ}, 50^{\circ}, 70^{\circ}\right)$ were dynamically investigated in that scenario at four discrete angles of attack $\left(\alpha=-5^{\circ}, 0^{\circ}\right.$, $5^{\circ}, 10^{\circ}$. Consequently, within the scope of this paper the comparison between the two sources for measuring the surface pressure focuses on those four spoiler deflection angles only.

Fig. 15 highlights the differences in the integrated force and moment coefficients when conducting the pressure integration solely with the data of the unsteady pressure transducers instead of the static pressure ports. It can be seen that the variations strongly depend on angle of attack and spoiler deflection. A very good agreement is achieved for the the lift coefficient up to the stall regime, Fig. 15(a). Beyond $\alpha=11^{\circ}$ minor deviations occur. For the case at $\delta=70^{\circ}$ a constant offset between the results is observed. The differences for the drag coefficient shown in Fig. 15(b) are more apparent. For angles of attack up to $\alpha=6^{\circ}$ the integration of the data from the unsteady pressure transducers yields a consistently lower drag than from 


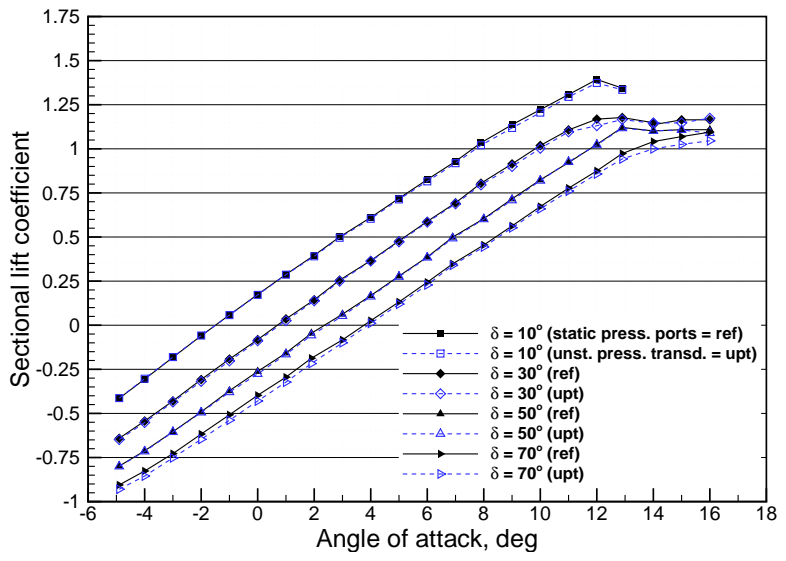

(a) Lift.

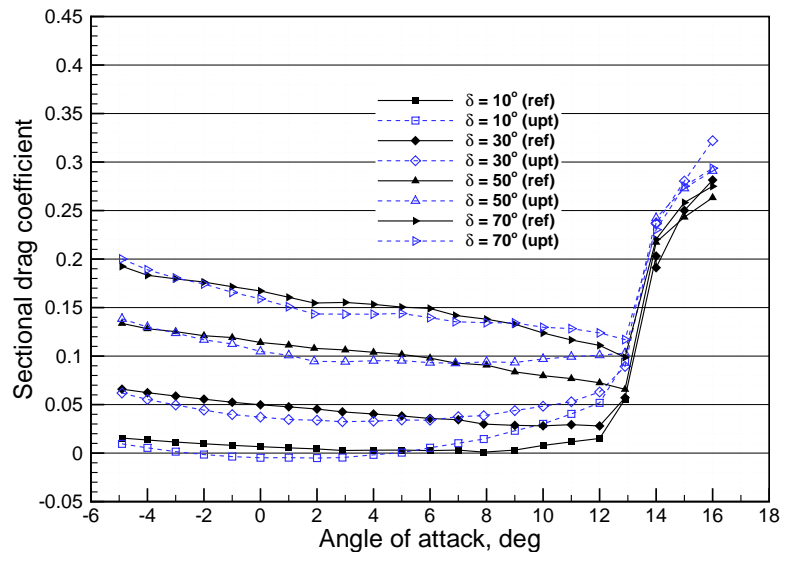

(b) Drag.

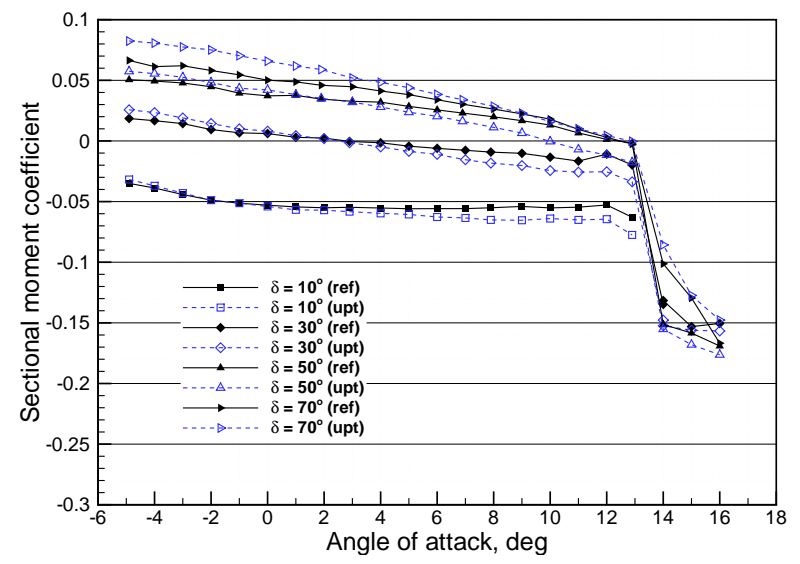

(c) Pitching moment.

Figure 15. Influence of the pressure integration on the integrated coefficients when using the unsteady pressure transducers instead of the static pressure ports.

the static taps. The negative drag coefficients observed at $\delta=10^{\circ}$ when using the data from the unsteady transducers is attributed to the fact that the friction drag is neglected and probably due to the relatively low number of unsteady pressure transducers distributed along the airfoil. For the pitching moment, Fig. 15(c), a very consistent behavior is observed again, with more pronounced differences for very low or high angles of attack.

Nonetheless, depending on the combination of $\alpha$ and $\delta$ used in a given dynamic scenario it is now possible with these results to exactly determine in how far the data of the unsteady pressure transducers need to be corrected before they can be compared with the data from the static pressure ports used in cases with static spoiler deflections.

\section{IV.F. Influence of the Dynamic Spoiler Deflection on the Transient Lift}

Having dealt with static spoiler deflections so far, some exemplary results of the ramp-type spoiler deflections from scenario (1) are shown in the following. One of the key aspects consisted in gradually reducing the spoiler deflection time $t_{\text {deploy, }}$, or the reduced deployment time $t^{*}$ according to Eq. (1), and hence try to induce the lift overshoot known as adverse lift. The transient lift response for the four investigated deflection angles are shown in Fig. 16.

A look at the embedded figures in each diagram quickly reveals that we were able to induce an adverse lift at every spoiler angle for at least the highest deflection rate. In case of an deployment to $\delta=50^{\circ}$, Fig. 16(c), adverse lift even occurs at two deflection rates, with deployment times of $t^{*}=9.2$ and $t^{*}=14.3$. It is also noteworthy that at $\delta=70^{\circ}$, Fig. $16(\mathrm{~d})$, adverse lift is observed for $t^{*}=14.3$ as well. These latter 


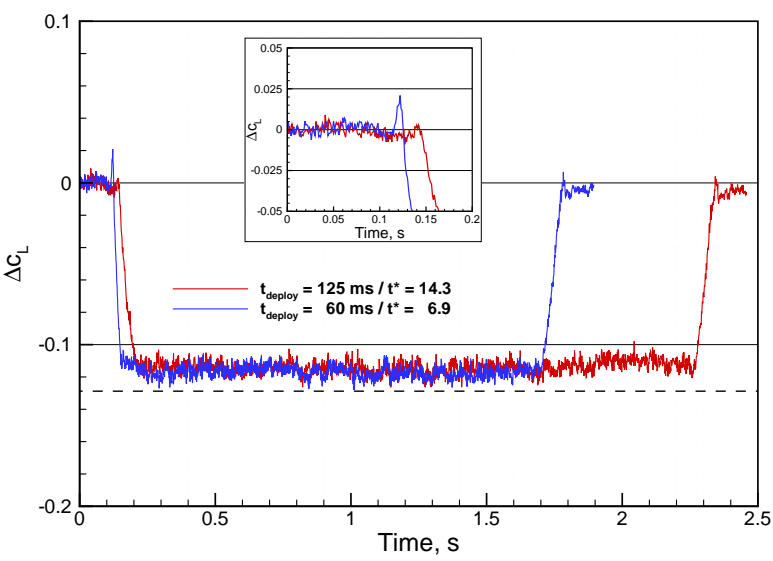

(a) $\delta=10^{\circ}$.

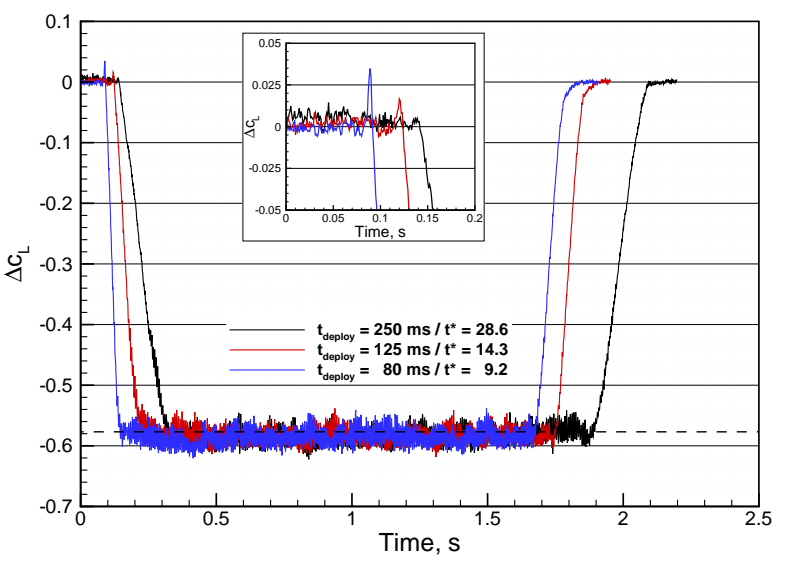

(c) $\delta=50^{\circ}$.

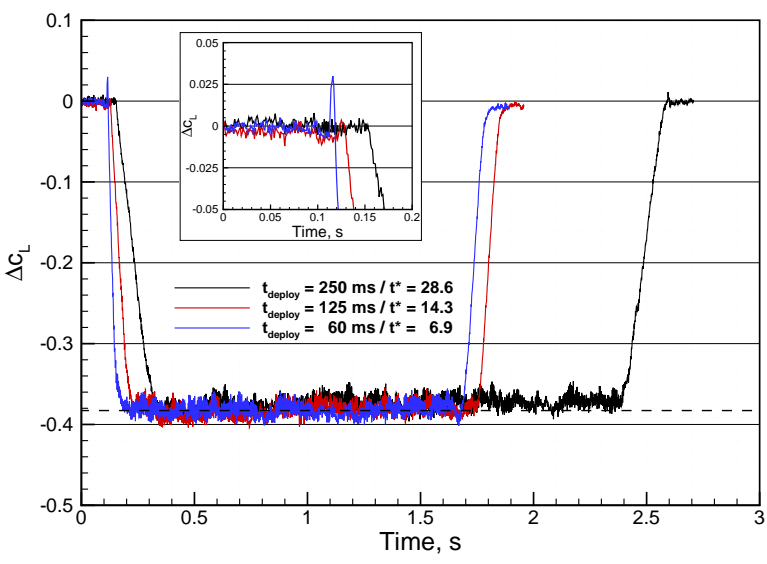

(b) $\delta=30^{\circ}$.

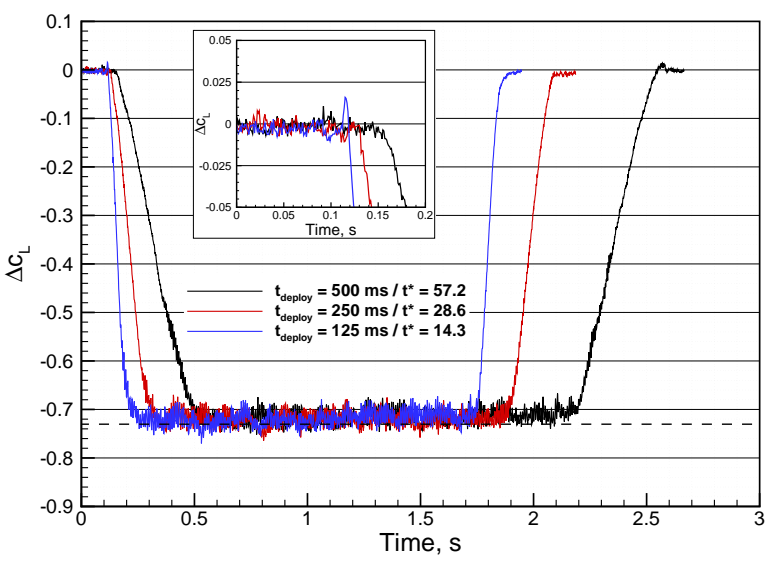

(d) $\delta=70^{\circ}$.

Figure 16. Influence of dynamic spoiler deflections at various spoiler deflection rates on the transient lift loss at $\alpha=0^{\circ}$, $M=0.2$. The horizontal dashed (- - ) line represents the lift loss for an equivalently deflected, static spoiler.

two results were unexpected, as according to the literature review no research work is known to the authors in which adverse lift was observed for deployment times $t^{*}>8-9$.

Figure 16 also reveals that the deflection rate has no influence on the lift loss and thus on the effectiveness of the spoiler. Apart from the case with a deflection to $\delta=10^{\circ}$, the dynamic spoiler effectiveness matches the static spoiler effectiveness very well. In case of the former the dynamic effectiveness is a little lower, Fig. 16(a).

The magnitude of the adverse lift, $\Delta C_{L a}$, is summarized in Table 3. While it appears appropriate to use the reduced time $t^{*}$ as the parameter to determine whether or not adverse lift occurs, it is not suited to judge on the magnitude of the adverse lift. As shown the shortest reduced time does not necessarily yield the highest $\Delta C_{L a}$. Further research will be conducted to understand the influence of the characteristic parameters such as deployment rate, reduced time, etc. on the adverse lift phenomenon.

Table 3. Adverse lift characteristics for dynamic spoiler deflections at $M=0.2$.

\begin{tabular}{ccccc}
\hline \hline Deployment angle & Deployment time & Reduced time $t^{*}$ & Avg. deployment rate & Adv. lift $\Delta C_{L a}$ \\
\hline $10^{\circ}$ & $60 \mathrm{~ms}$ & 6.9 & $167^{\circ} / \mathrm{s}$ & 0.0209 \\
\hline $30^{\circ}$ & $60 \mathrm{~ms}$ & 6.9 & $500^{\circ} / \mathrm{s}$ & 0.0299 \\
\hline $50^{\circ}$ & $125 \mathrm{~ms}$ & 14.3 & $400^{\circ} / \mathrm{s}$ & 0.0166 \\
& $80 \mathrm{~ms}$ & 9.2 & $625^{\circ} / \mathrm{s}$ & 0.0348 \\
\hline $70^{\circ}$ & $125 \mathrm{~ms}$ & 14.3 & $560^{\circ} / \mathrm{s}$ & 0.0159 \\
\hline \hline
\end{tabular}




\section{Conclusion and Outlook}

The present paper gives an overview on the motivation, setup and exemplary results of an experimental investigation on the steady and unsteady aerodynamic behavior of a configuration with static and dynamic spoiler. The objective of this study consisted in gaining a better insight into the characteristic flow features for applications with deployed control surfaces, in particular for a dynamic spoiler. Moreover, the data of this campaign and an already completed second campaign, in which unsteady PIV was applied to visualize the transient flow field, will be used to validate the DLR TAU RANS flow solver for applications with dynamic control surfaces.

For this purpose, a new wind tunnel model was developed by DLR and tested in clean configuration, with various static spoiler deflections and two dynamic spoiler deployment scenarios. This vast amount of validation data enable DLR to assess the impact of parameters like angle of attack, deployment angle or deployment rate on the aerodynamic behavior of a spoiler.

The adverse effects during rapid spoiler deployment, exemplarily shown in terms of transient lift response, will be further studied in more detail. In addition, the PIV data of the second campaign will be used to gain a more detailed insight into the relevant flow physics for static and dynamic spoiler deflections. Based on the presented results, further work has already been planned in a successor project: while the Mach and Reynolds number effects will be investigated numerically, the DLR-F15DS model will be used to experimentally investigate sweep effects on the dynamic 3D spoiler in the low-speed regime.

\section{Acknowledgments}

The authors would like to thank D. Peters, M. Gülzow, S. Seibt, M. Marx and M. Gehrke from the DNW-NWB wind tunnel team and J. Bruns from the DLR Institute of Aerodynamics and Flow Technology for their great support in preparing and conducting the wind tunnel tests. Special thanks as well go to J. Wild, same institute, for his continuous support and technical advice during the preparation phase.

\section{References}

\footnotetext{
${ }^{1}$ Becker, K. and Vassberg, J., "Numerical Aerodynamics in Transport Aircraft Design," Notes on Numerical Fluid Mechanics and Multidisciplinary Design, edited by E.-H. Hirschel and E. Krause, Vol. 100, Springer, 2009, pp. $209-220$.

${ }^{2}$ Rossow, C.-C. and Cambier, L., "European Numerical Aerodynamics Simulation Systems," Notes on Numerical Fluid Mechanics and Multidisciplinary Design, edited by E.-H. Hirschel and E. Krause, Vol. 100, Springer, 2009, pp. $189-208$.

${ }^{3}$ Vassberg, J. C., Tinoco, E. N., Mani, M., Rider, B., Zickuhr, T., et al., "Summary of the Fourth AIAA Computational Fluid Dynamics Drag Prediction Workshop," Journal of Aircraft, Vol. 51, No. 4, July-August 2014, pp. 1070-1089.

${ }^{4}$ Tinoco, E. N., Bogue, D. R., Kao, T.-J., Yu, N. J., Li, P., and Ball, D. N., "Progress Toward CFD for Full Flight Envelope," The Aeronautical Journal, Vol. 109, No. 1100, October 2005, pp. 451-460.

${ }^{5}$ Geisbauer, S., "Numerical Spoiler Wake Investigations at the Borders of the Flight Envelope," AIAA Paper 2011-3811, $29^{\text {th }}$ AIAA Applied Aerodynamics Conference, June 2011.

${ }^{6}$ Flaig, A., "Airbus A380: Solutions to the Aerodynamic Challenges of Designing the World's Largest Passenger Aircraft," Presentation, Royal Aeronautical Society, Hamburg, http://www.fzt.haw-hamburg.de/pers/Scholz/dglr/hh/text_2008_01_ 30_A380.pdf, retrieved October 2016, 2008.

${ }^{7}$ Kroll, N., Abu-Zurayk, M., Dimitrov, D., Franz, T., Führer, T., et al., "DLR Project Digital-X: Towards Virtual Aircraft Design and Flight Testing Based on High-Fidelity Methods," CEAS Aeronautical Journal, Vol. 7, No. 1100, December 2015, pp. $3-27$.

${ }^{8}$ Sensburg, O., Becker, J., Lusebrink, H., and Weiss, F., "Gust Load Alleviation on Airbus A300," ICAS Paper 82-2.2.1, International Council of the Aeronautical Sciences, August 1982.

${ }^{9}$ Payne, B. W., "Designing a Load Alleviation System for a Modern Civil Aircraft," ICAS Paper 86-5.2.3, International Council of the Aeronautical Sciences, September 1986.

${ }^{10}$ König, R., Hahn, K.-U., and Winter, J., "Advanced Gust Management Systems - Lessons Learned and Perspectives," AGARD-CP-560 paper 10, AGARD Conference Proceedings, January 1995.

${ }^{11} \mathrm{Xu}$, J. and Kroo, I., "Aircraft Design with Maneuver and Gust Load Alleviation," AIAA Paper 2011-3180, 29 ${ }^{\text {th }}$ AIAA Applied Aerodynamics Conference, June 2011.

${ }^{12}$ Pusch, M., Knoblach, A., and Kier, T., "Integrated Optimization of Ailerons for Active Gust Load Alleviation," IFASD Paper 2015-199, International Forum on Aeroelasticity and Structural Dynamics (IFASD), June 2015.

${ }^{13}$ Fage, A. and Johansen, F. C., "On the Flow of Air Behind an Inclined Flat Plate of Infinite Span," Proceedings of the Royal Society of London, Series A, Vol. 116, No. 773, September 1927, pp. 170-197.

${ }^{14}$ Mack, M. D., Seetharam, H. D., Kuhn, W. G., and Bright, J. T., "Aerodynamics of Spoiler Control Devices," AIAA Paper 1979-1873, AIAA Aircraft Systems and Technology Meeting, August 1979.

${ }^{15}$ Ayoub, A., Satynarayana, B., and Karamcheti, K., "Unsteady Flow Patterns Associated with Spoiler Control Devices," AIAA Paper 1982-0127, $20^{t h}$ AIAA Aerospace Sciences Meeting, January 1982.
} 
${ }^{16}$ McLachlan, B. G., Karamcheti, K., Ayoub, A., and Hadjidakis, G., "A Study of the Unsteady Flow Field of an Airfoil with Deflected Spoiler," AIAA Paper 1983-2131, 10 ${ }^{\text {th }}$ AIAA Atmospheric Flight Mechanics Conference, August 1983.

${ }^{17}$ McLachlan, B. G. and Karamcheti, K., "An Experimental Study of Airfoil-Spoiler Aerodynamics," JIAA-TR 52, Joint Institute for Aeronautics and Acoustics, Stanford University, April 1984.

${ }^{18}$ Lee, C. S. and Bodapati, S., "Experimental Investigations of the Flowfield of an Airfoil with Spoiler," AIAA Journal, Vol. 25, No. 4, November 1987, pp. 1411-1416.

${ }^{19}$ Wentz, Jr., W., Ostowari, C., and Seetharam, H. C., "Effects of Design Variables on Spoiler Control Effectiveness, Hinge Moments and Wake Turbulence," AIAA Paper 1981-0072, $19^{\text {th }}$ AIAA Aerospace Sciences Meeting, January 1981.

${ }^{20}$ Siddalingappa, S. R. and Hancock, G. J., "An Introduction to the Aerodynamics of Spoilers," Paper QMC EP-1034, Department of Aeronautical Engineering, Queen Mary College, London, August 1980.

${ }^{21}$ Hancock, G. J., "Dynamic Effects of Controls," AGARD-R-711 paper 4, AGARD Special Course on Aerodynamic Characteristics of Controls, July 1995.

${ }^{22}$ Mabey, D. G., "Experimental Methods to Determine Control Effectiveness in Wind Tunnels," AGARD-R-711 paper 5, AGARD Special Course on Aerodynamic Characteristics of Controls, July 1995.

${ }^{23}$ Consigny, H., Gravelle, A., and Molinaro, R., "Aerodynamic Characteristics of a Moving Two-Dimensional Spoiler in Subsonic and Transonic Flow," Journal of Aircraft, Vol. 21, No. 9, September 1984, pp. 687-683.

${ }^{24}$ Kalligas, K., "The Dynamic Characteristics of Two-Dimensional Spoilers at Low Speeds," Ph.d. thesis, Department of Aeronautical Engineering, University of Bristol, December 1986.

${ }^{25}$ Costes, M., Gravelle, A., Philippe, J. J., Vogel, S., and Triebstein, H., "Investigation of Unsteady Subsonic Spoiler and Flap Aerodynamics," Journal of Aircraft, Vol. 24, No. 9, September 1987, pp. 629-637.

${ }^{26}$ Nelson, C. F., Koga, D. J., and Eaton, J. K., "Unsteady, Separated Flow Behind an Oscillating, Two-Dimensional Spoiler," AIAA Journal, Vol. 28, No. 5, May 1990, pp. 845-852.

${ }^{27}$ Yeung, W. W. H., Xu, C., and Gu, W., "Reduction of Transient Adverse Spoiler Effects," Journal of Aircraft, Vol. 34, No. 4, July-August 1990, pp. 479-484.

${ }^{28}$ Mineck, R. E., "Reynolds Number Effects on the Performance of Ailerons and Spoilers," AIAA Paper 2001-0908, 39 ${ }^{t h}$ AIAA Aerospace Sciences Meeting and Exhibit, January 2001.

${ }^{29}$ NATO-RTO, "Verification and Validation Data for Computational Unsteady Aerodynamics," RTO Technical Report 26, Applied Vehicle Technology Panel (AVT) Task Group AVT-010, October 2000.

${ }^{30}$ Wild, J., Pott-Polenske, M., and Nagel, B., "An Integrated Design Approach for Low-Noise Exposing High-Lift Devices," AIAA Paper 2006-2843, $3^{\text {rd }}$ AIAA Flow Control Conference, June 2006.

${ }^{31}$ Garner, H. C., Rogers, E. W., Acum, W. E., and Maskell, E. C., "Subsonic Wind Tunel Wall Corrections," AGARDograph 109, Advisory Group for Aerospace Research and Development (AGARD), October 1966.

${ }^{32}$ Maskell, E. C., "A Theory of the Blockage Effects on Bluff Bodies and Stalled Wings in a Closed Wind Tunnel," ARC R \& M 3400, Royal Aircraft Establishment (RAE), November 1963. 